\title{
Surrogate measures of insulin resistance: does one size fit all?
}

\author{
Donald C. Simonson
}

Received: 14 October 2014 / Accepted: 3 November 2014 /Published online: 22 November 2014

(C) Springer-Verlag Berlin Heidelberg 2014

\begin{abstract}
Insulin resistance and a relative deficiency in insulin secretion are the major pathophysiological features of impaired glucose tolerance and type 2 diabetes. Although the euglycaemic-hyperinsulinaemic clamp and the hyperglycaemic clamp are the 'gold standards', respectively, for measuring these metabolic defects, there has been an active search during the past few decades for simpler and less expensive surrogate measures of insulin resistance that can be applied more globally in epidemiological studies or large clinical trials. These surrogate markers primarily rely on plasma insulin and glucose levels measured either in the fasting state or after an oral glucose challenge. Although many of these surrogate measures correlate well with the clamp (with $r$ values frequently as high as 0.60 0.70 ) within racially and ethnically homogeneous populations, it is not clear how well they can be used to compare insulin action across different groups. In this issue of Diabetologia, Ahuja et al (DOI: 10.1007/s00125-014-3414-6) have used these methods to compare insulin resistance in a white population in the USA with a Japanese population in Japan. The relative merits and drawbacks of applying these techniques in different populations are discussed.
\end{abstract}

Keywords Asian - Disposition index - Ethnicity . HOMA · Hyperglycaemic clamp - Hyperinsulinaemic clamp - Impaired glucose tolerance - Insulin resistance . Race . Type 2 diabetes

\section{Abbreviations \\ DI Disposition index \\ HOMA- $\beta \%$ HOMA of beta cell function}

\section{C. Simonson $(\square)$}

Division of Endocrinology, Diabetes and Hypertension,

Brigham and Women's Hospital, Harvard Medical School,

221 Longwood Avenue, Boston, MA 02115, USA

e-mail: dsimonso@hsph.harvard.edu
IFG Impaired fasting glucose

IGT Impaired glucose tolerance

VAT Visceral adipose tissue

Insulin resistance and a relative deficiency of glucosestimulated insulin secretion are the two pathophysiological hallmarks of impaired glucose tolerance (IGT) and type 2 diabetes. Thus, it is not surprising that much of the clinical metabolic research conducted during the past several decades has been dedicated to developing methods to measure these abnormalities, and using them to study the underlying tissue and cellular mechanisms responsible.

Over 35 years have passed since the publication of the two pre-eminent methods for studying insulin resistance and secretion, namely, the euglycaemic-hyperinsulinaemic clamp and the hyperglycaemic clamp [1]. Despite the passage of time, these techniques are still considered the 'gold standard', and with good reason. They are the only widely used techniques that effectively interrupt the normal homeostatic feedback mechanisms that exist between tissue glucose uptake, insulin secretion and the plasma glucose concentration, thus enabling the investigator to study each variable independently while controlling the others. Unfortunately, clamp studies require large quantities of money, time, technical expertise and willing patient volunteers, all of which are in short supply.

To overcome the practical limitations of performing clamp studies, investigators have developed many other techniques that rely on laboratory and clinical measures that are easier to perform and less expensive. These surrogate measures of insulin resistance have become widely accepted, especially in the fields of epidemiology and large clinical trials, but their limitations have also been widely noted. They typically rely on the measurement of glucose and insulin either in the fasting state (e.g. HOMA-IR [2] or Quantitative Insulin Sensitivity Check Index [QUICKI] [3]) or after oral glucose ingestion 
(e.g. the insulin sensitivity indices of Matsuda [4] or Stumvoll [5]). However, all of these methods depend on the preservation of a normal feedback mechanism between plasma glucose levels and insulin secretion, i.e. a metabolic state no worse than impaired fasting glucose (IFG) or IGT. Most of these methods become quite imprecise or cannot be used at all in the patient population we would most like to study, i.e. those with type 2 diabetes. An excellent meta-analysis of the validation methods of these surrogate measures was recently published by Otten et al [6] (with accompanying commentary [7]), and the reader is referred to this article for a thorough evaluation of the different methods.

Other surrogate measures of insulin resistance that do not involve direct measurement of glucose or insulin levels have also gained popularity. Anthropometric measures such as waist circumference or waist/hip ratio are easy to obtain and also predictive of disordered glucose homeostasis [8]. Fasting plasma triacylglycerol level (or the ratio of triacylglycerol to HDL-cholesterol) is perhaps the most commonly measured routine clinical laboratory test that correlates well with the severity of insulin resistance. Of note, these measures are also key components of our current definition of the metabolic syndrome. Inflammatory markers such as C-reactive protein or adipokines (including leptin or adiponectin) have also been studied for their general applicability to identifying patients with insulin resistance.

Most of the surrogate measures of insulin resistance, like the clamp technique itself, have been developed in the predominantly white populations of Western Europe and North America. In fact, of the 120 publications included in the validation meta-analysis referenced above [6], $41 \%$ of the studies were performed in Western Europe and 33\% in the USA or Canada. However, as reported in publications from the WHO and the International Diabetes Federation (IDF) on the impending epidemic of type 2 diabetes $[9,10]$, most new cases of diabetes are projected to occur in the economically developing populations of South Asia (India, Pakistan and Bangladesh), East Asia (China), the Middle East and North Africa, and Central and South America. Given the challenges we will face in studying the pathophysiology of diabetes and the relative merits of new therapies in these developing regions with relatively limited financial and medical resources, it is important to address the practical and theoretical issues involved in the application of surrogate measures of insulin resistance in these diverse populations.

Most surrogate measures of insulin resistance have been well studied within specific racial or ethnic groups and, fortunately, most studies have found that they correlate well with clamp studies or other clinical or laboratory measures of insulin resistance [6]. However, their use as comparative measures between groups of different racial and ethnic origin is more problematic. The article by Ahuja et al [11] in this issue of Diabetologia attempts to address some of these issues by comparing HOMA-IR, HOMA of beta cell function (HOMA- $\beta \%$ ) and the disposition index (DI) in 298 white men in the USA with 294 Japanese men in Japan to determine the relative contribution of visceral adipose tissue (VAT) and other metabolic covariates to differences in insulin sensitivity and secretion. They report that white men have higher levels of all three surrogate measures after correcting for differences in VAT, and this difference persists after correcting for BMI, fasting glucose, C-reactive protein and a variety of lifestyle factors.

This is one of the few studies in this field to have compared two different racial groups, each studied in their respective homelands, while using a standardised protocol with laboratory measures performed and radiological images interpreted at a central location. Most other studies have compared immigrant populations or their descendants with the predominant resident population, e.g. comparing South Asian populations in the USA or Western Europe with the predominantly white inhabitants of those countries. One approach is not clearly preferable over the other, as the former must attempt to separate differences owing to genetic factors from inextricably linked dietary and other cultural factors, while the latter must account for the inevitable blending of dietary and lifestyle factors that occurs when members of one racial or ethnic group immigrate to a country with a different culture.

Ahuja et al [11] employ surrogate measures of insulin resistance (HOMA-IR), insulin secretion (HOMA- $\beta \%$ ) and a somewhat unconventional application of the DI based on the two fasting measures. The DI was originally based on measures of insulin sensitivity and secretion in the stimulated state, e.g. during a clamp study or after an oral or intravenous glucose challenge. The use of fasting surrogates for both measures, particularly when comparing two groups as different as the two in this publication, should be undertaken with caution. Because the correlation ( $r$ value) between HOMA-IR and clamp-based measures of insulin resistance is typically 0.6 , the variance explained by HOMA-IR $\left(r^{2}\right)$ would only be about $36 \%$ [6]. Furthermore, the correlation between HOMA- $\beta \%$ and stimulated measures of insulin secretion (hyperglycaemic clamp or IVGTT) is not well studied, but is unlikely to be much better. This would introduce substantial variability (resulting from measurement error, potential measurement bias or sampling variability) into the relationship described by DI (HOMA- $\beta \% / H O M A-I R)$. This increases the probability of failing to find a significant relationship when one truly exists (type II statistical error).

A similar line of reasoning applies when the authors examine the relationship between these surrogate measures of insulin action while attempting to adjust for many of the covariates listed in Table 1 of the article [11]. As is apparent from the table, there are highly significant differences between groups for many of the important covariates. This is not surprising given the differences in anthropometrics, dietary 
Table 1 Physiological and clinical characteristics of insulin resistance and its consequences in major racial and ethnic populations projected to have the highest future risk of diabetes

\begin{tabular}{llllll}
\hline Physiological or clinical measure & \multicolumn{5}{l}{ Racial/ethnic population } \\
\cline { 2 - 6 } & South Asian & East Asian & Black & Middle Eastern & Central/South American/Hispanic (USA) \\
\cline { 2 - 6 } Insulin resistance & +++ & ++ & +++ & +++ & ++ \\
$\uparrow$ Insulin secretion & +++ & + & ++ & ++ & ++ \\
$\uparrow$ BMI & ++ & + & +++ & ++ & ++ \\
$\uparrow$ Waist or VAT & +++ & ++ & ++ & ++ & ++ \\
$\uparrow$ TG and/or $\downarrow$ HDL-C & +++ & + & + & ++ & ++ \\
$\uparrow$ BP & ++ & ++ & +++ & ++ & ++ \\
CVD risk & +++ & ++ & +++ & ++ & ++ \\
Type 2 diabetes risk & +++ & ++ & ++ & +++ & ++ \\
\hline
\end{tabular}

Plus $(+)$ signs indicate greater relative strength or presence of the physiological or clinical abnormality in each population relative to the other four. CVD, cardiovascular disease; TG, triacylglycerol; HDL-C, HDL cholesterol

patterns and cultural habits between Japanese and white American populations. For example, given the highly significant difference in BMI between groups, there may not be enough heavier Japanese (or thinner white Americans) in the data to satisfy the assumption of a linear relationship across groups. Thus, if the relationship between BMI and VAT is not linear within one or both populations, it would not be correct to extrapolate the assumption of linearity into the range of BMI that does not apply to the other group. One must also consider whether surrogate measures of insulin sensitivity and secretion, given all of their limitations, have an appropriate degree of precision when compared with the high degree of accuracy that laboratory and radiological measures inherently possess. Again, this increases the variability in the association being studied, causing one to possibly miss a significant relationship rather than falsely drawing a positive conclusion.

Many studies have shown that within a particular racial or ethnic group, including East Asian, South Asian, Middle Eastern, Hispanic/Latino, and black/African, surrogate measures based on fasting or post-glucose challenge generally have correlations with the clamp that are similar to those seen in white Europeans $(r=0.50-0.70)$ [6]. However, this may belie differences in the underlying physiology.

Although there have been relatively few well-controlled studies comparing the demographic, metabolic, biochemical and anthropometric characteristics of multiple racial and ethnic groups under comparable conditions [12], it is possible to glean some general trends from the studies that have been published within individual groups. In all populations there is a decrease in insulin sensitivity and a relative deficiency of compensatory insulin secretion that leads to progressive deterioration from normal glucose tolerance to IGT and type 2 diabetes. However, the relative predominance of one defect, and the clinical importance of other associated pathophysiological and laboratory abnormalities, often differ among groups (Table 1).
In South Asian populations the impairment in insulin action appears to be the predominant feature, associated with a vigorous, albeit still inadequate, insulin response. This is accompanied by increased VAT as measured either directly by radiographic means or indirectly by waist circumference. The classic diabetic dyslipidaemia of increased triacylglycerols and low HDL-cholesterol and an increase in premature cardiovascular disease are also prominent features.

In East Asian populations, however, an insufficient beta cell response to worsening insulin resistance appears consistently in the published literature. Although East Asians tend to have smaller body habitus compared with most other major racial and ethnic groups, they are nonetheless characterised by a relative increase in waist circumference and central adiposity. This is reflected in the smaller waist circumference required for the diagnosis of the metabolic syndrome in Asian populations. Of note, a number of studies from East Asian countries also report that abnormal liver function tests are a close correlate of insulin resistance, although it is not clear whether this represents selective reporting due to the interest of the investigator, or a true increase in non-alcoholic fatty liver disease or subclinical hepatitis $\mathrm{C}$, both of which are associated with insulin resistance and diabetes.

In contrast, among Central and South American populations (predominantly studied in the Hispanic or Latino population in the USA), insulin resistance is accompanied by a robust insulin secretory response. Increased waist circumference and dyslipidaemia are also frequently present. Among black/African populations, again primarily studied in the USA, similar abnormalities in insulin resistance, defective insulin secretion and increased waist circumference are reported. However, there is a notable increase in the association of insulin resistance with hypertension, congestive heart failure and early onset coronary artery disease, with less evidence of the classic dyslipidaemia associated with insulin resistance in other populations. 
Thus, although surrogate measures of insulin resistance may be valid within a population, one must be careful in using them in comparisons between racial and ethnic groups where different physiological mechanisms may be dominant. For example, among patients with similar HOMA-IR values, a hypothetical new pharmacological treatment or lifestyle intervention that improves insulin secretion might be more effective in East Asians, while a medication that targets VAT and dyslipidaemia might be preferable in South Asians, and one that also targets hypertension and vascular disease might be more desirable in black/African populations. Surrogate measures can play a valuable role in generating hypotheses from epidemiological data or for directing clinical investigators toward mechanistic studies based on results from clinical trials, but to understand the basic physiology of insulin resistance, insulin secretion and the downstream pathological consequences of the metabolic syndrome, one must still rely on the more sophisticated methodology and controlled experimental environment afforded by the insulin clamp or similar interventional research techniques.

Duality of interest The author declares that there is no duality of interest associated with this manuscript.

Contribution statement The author was the sole contributor to this paper

\section{References}

1. DeFronzo RA, Tobin JD, Andres R (1979) Glucose clamp technique: a method for quantifying insulin secretion and resistance. Am J Physiol 237:E214-E223
2. Matthews DR, Hosker JP, Rudenski AS, Naylor BA, Treacher DF, Turner RC (1985) Homeostasis model assessment: insulin resistance and beta-cell function from fasting plasma glucose and insulin concentrations in man. Diabetologia 28:412-419

3. Katz A, Nambi SS, Mather K et al (2000) Quantitative insulin sensitivity check index: a simple, accurate method for assessing insulin sensitivity in humans. J Clin Endocrinol Metab 85:24022410

4. Matsuda M, DeFronzo RA (1999) Insulin sensitivity indices obtained from oral glucose tolerance testing: comparison with the euglycemic insulin clamp. Diabetes Care 22:1462-1470

5. Stumvoll M, Mitrakou A, Pimenta W et al (2000) Use of the oral glucose tolerance test to assess insulin release and insulin sensitivity. Diabetes Care 23:295-301

6. Otten J, Ahrén B, Olsson T (2014) Surrogate measures of insulin sensitivity vs the hyperinsulinaemic-euglycaemic clamp: a metaanalysis. Diabetologia 57:1781-1788

7. Petrie JR (2014) Evidence-based estimation of insulin resistance. Diabetologia 57:1743-1745

8. Alberti KG, Eckel RH, Grundy SM et al (2009) Harmonizing the metabolic syndrome: a joint interim statement of the International Diabetes Federation Task Force on Epidemiology and Prevention; National Heart, Lung, and Blood Institute; American Heart Association; World Heart Federation; International Atherosclerosis Society; and International Association for the Study of Obesity. Circulation 120: $1640-1645$

9. Wild S, Roglic G, Green A, Sicree R, King H (2004) Global prevalence of diabetes: estimates for the year 2000 and projections for 2030. Diabetes Care 27:1047-1053

10. Whiting DR, Guariguata L, Weil C, Shaw J (2011) IDF diabetes atlas: global estimates of the prevalence of diabetes for 2011 and 2030. Diabetes Res Clin Pract 94:311-321

11. Ahuja V, Kadowaki T, Evans RW et al (2014) Comparison of HOMA-IR, HOMA-B\% and disposition index between US white men and Japanese men in Japan: the ERA JUMP study. Diabetologia. doi:10.1007/s00125-014-3414-6

12. Kodama K, Tojjar D, Yamada S, Toda K, Patel CJ, Butte AJ (2013) Ethnic differences in the relationship between insulin sensitivity and insulin response: a systematic review and meta-analysis. Diabetes Care 36:1789-1796 